Y.-H. Chu, N.B. Suntzeff, J.E. Hesser, and D.A. Bohlender, eds.

\title{
The Low End of the Initial Mass Function for the R136 Cluster in the LMC
}

\author{
M. Sirianni ${ }^{1}$
}

Johns Hopkins University, Baltimore, MD 21218, USA

A. Nota ${ }^{2}$, C. Leitherer, M. Clampin

Space Telescope Science Institute, Baltimore, MD 21218, USA

G. De Marchi

European Southern Observatory, Garching bei München, Germany

Multiple images of the R136 cluster in the Large Magellanic Cloud taken with the HST/WFPC2 and the F555W and F814W filters were combined to obtain very deep images of the cluster, with total exposure times of 1240 and $760 \mathrm{sec}$ respectively. The objective of the study was to extend the luminosity function below the limit of $2.8 \mathrm{M}_{\odot}$ published by Hunter et al. $(1995,1996)$.

PSF fitting photometry was performed, with the conservative threshold set to discard all stars with an associated error larger than $0.2 \mathrm{mag}$ in both filters. We then found 1604 stars common to both filters down to a limiting magnitude of 24.7 , or $\sim 1 \mathrm{mag}$ deeper than the data published by Hunter et al. $(1995,1996)$. We generated a dereddened, color-magnitude diagram (CMD) by adopting the reddening law used by Hunter et al. (1995). Two distinct populations appear in Fig. 1 segregated in color at $\left(m_{F 555 W}-m_{F 814 W}\right)_{0} \simeq 0.5$.

The observed red population is very well tracked by PMS isochrones of Bernasconi (1996), and most likely consists of low mass stars (down to $0.8 \mathrm{M}_{\odot}$ ) still approaching the main sequence. Their ages are found to be between 1 and $10 \mathrm{Myr}$, in very good agreement with previous age determinations by Hunter et al. (1995) (4 Myr) and De Marchi et al. (1993) (3 Myr).

In order to locate the stars on the H-R diagram we converted the magnitudes and colors into the $\log \mathrm{T}_{\text {eff }} v s \log \mathrm{L} / \mathrm{L}_{\odot}$ plane. We performed the completeness test for our data defining four regions surrounding the R136 cluster excluding the inner region of $4^{\prime \prime}$. The four regions have different characteristics in terms of gas/dust contamination and crowding.

The Initial Mass Function (IMF), $\xi$, is defined as the number of star per logarithmic mass interval per unit area and its slope is given by $\Gamma=d(\log \xi) / d(\log M)$ where the standard IMF (Salpeter 1955) has a slope $\Gamma=-1.35$. In the case of the very young R136 cluster with coeval star formation, we can assume that the observed MF is the IMF. In order to derive the IMF of R136, we counted the number of stars falling between each pair of tracks in the HR diagram, corrected

\footnotetext{
${ }^{1}$ CISAS - University of Padova

${ }^{2}$ Affiliated to ESA,Astrophysics Division,Space Science Department of ESA.
} 

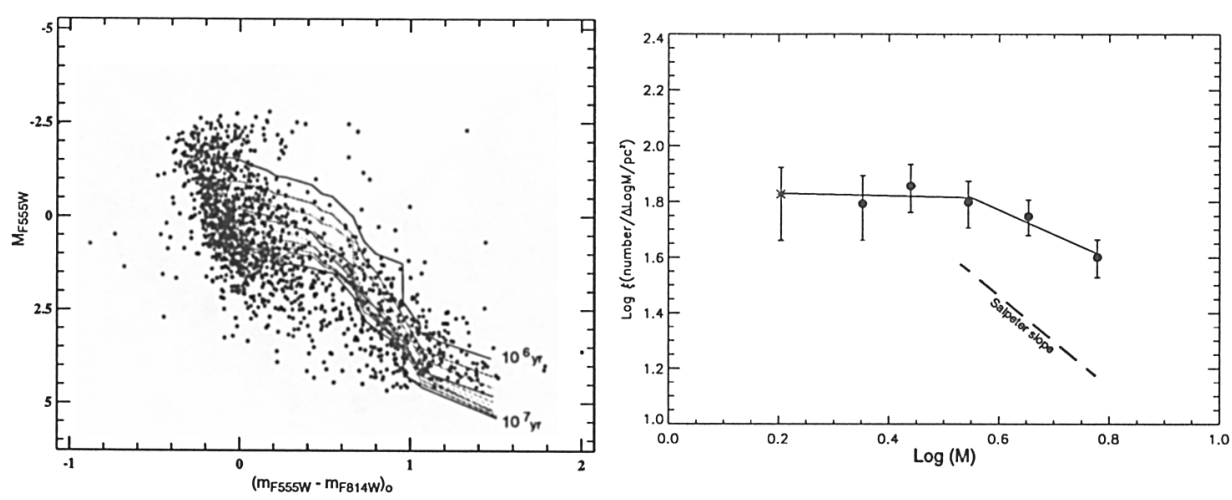

Figure 1. (left) Dereddened CMD of R136 with PMS isochrones. (right) The IMF of R136.

the number with the completeness factor and normalized the result to the width of the mass range spanned by the tracks and to the area of the observed field.

At masses of $0.8-2.75 \mathrm{M}_{\odot}$, we adopted the PMS tracks by Bernasconi (1996), while at higher masses $\left(3-7 \mathrm{M}_{\odot}\right)$ we used the standard evolutionary tracks of Schaller et al. (1992). In the overlap region around $3 \mathrm{M}_{\odot}$ both sets of tracks agree within the statistical errors. We used the two regions surrounding the core of R136 with the best completeness to construct the final IMF. Two different slopes are distinguishable in the IMF profile (Fig. 1): for stars in the mass range 3-7 $\mathrm{M}_{\odot}$ the data points are well fitted by a slope with $\Gamma=-0.88 \pm 0.06$, while at lower masses $\left(0.8-2.75 \mathrm{M}_{\odot}\right)$ the IMF profile flattens out, with a derived slope $\Gamma$ $=-0.04 \pm 0.06$. A similar effect, i.e., a deficiency of stars in the $1-2 \mathrm{M}_{\odot}$ range, is also observed by Hillenbrand (1997) in the Orion Nebula Cluster, although in that case the plateau is followed first by a steep increase between $0.5 \mathrm{M}_{\odot}$ and $0.2 \mathrm{M}_{\odot}$ and then by a clear drop all the way down to the H-burning limit. Although there are several examples of a flat IMF for stars less massive than $\sim 1 \mathrm{M}_{\odot}$ (e.g., Comeron, Rieke, \& Rieke (1997) in NGC 2024), only in $\rho$ Oph have Williams et al. (1995) found a flat IMF above $1 \mathrm{M}_{\odot}$. The question as to whether the flattening that we observe in R136 is characteristic of this cluster or a general feature remains to be answered.

\section{References}

Bernasconi, G. 1996 A\&AS, 120, 57

Comeron, F., Rieke, G. H., \& Rieke, M.J. 1996, ApJ, 473, 294

De Marchi, G., et al. 1993 ApJ, 419, 658

Hillenbrand, L.A. 1997, AJ, 113.1733

Hunter, D.A., et al. 1995, ApJ, 448, 179

Hunter, D.A., et al. 1996, ApJ, 459, L27

Schaller, G., Shaerer, D., Meynet, G., \& Maeder, A. 1992, A\&AS 96, 269

Williams, D.M., et al. 1995, ApJ, 454, 144 

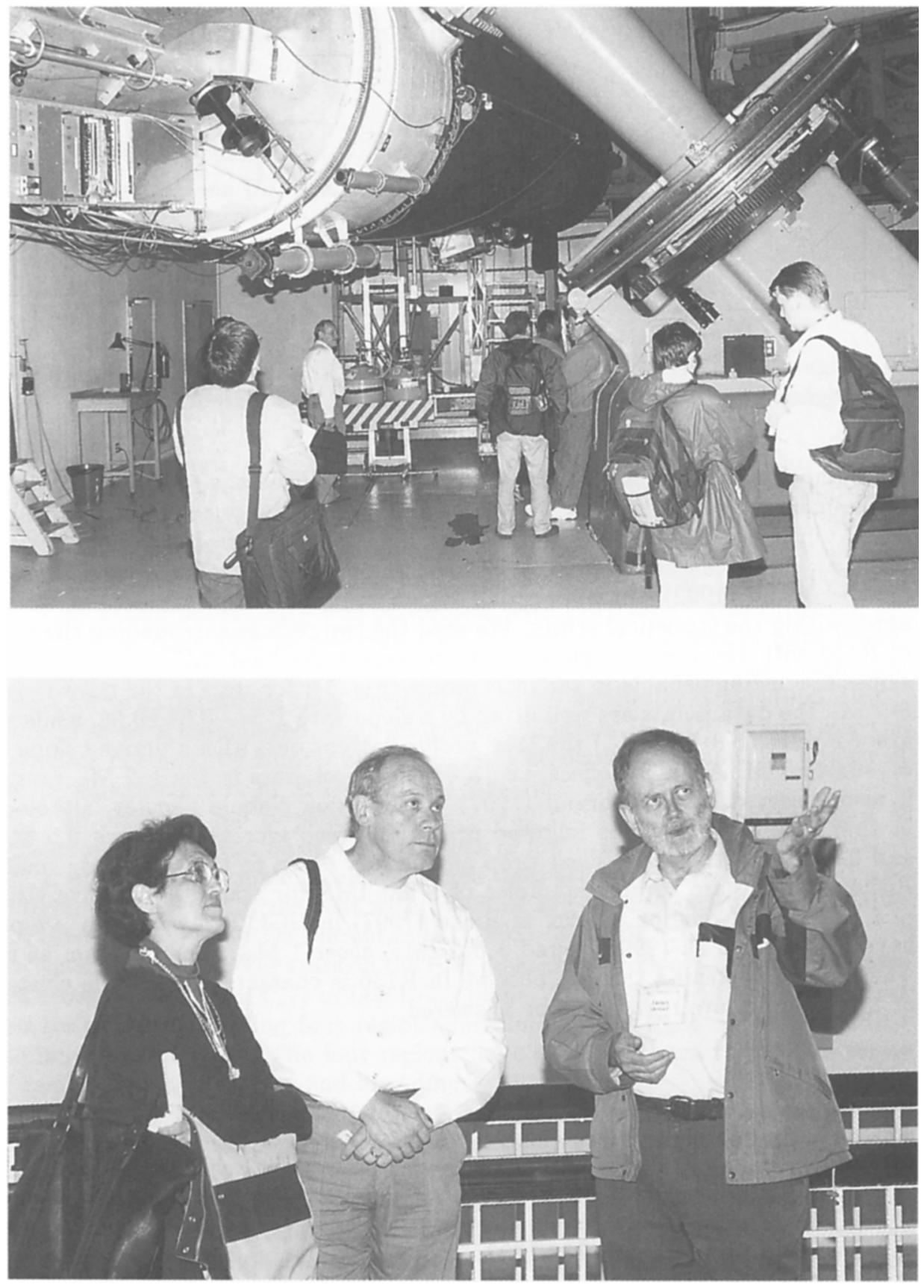

(Bottom) Mary and Evangelos Kontizas listen while Jim Hesser comments on the difficulty of observing the Magellanic Clouds from Victoria with the Plaskett Telescope (top). 